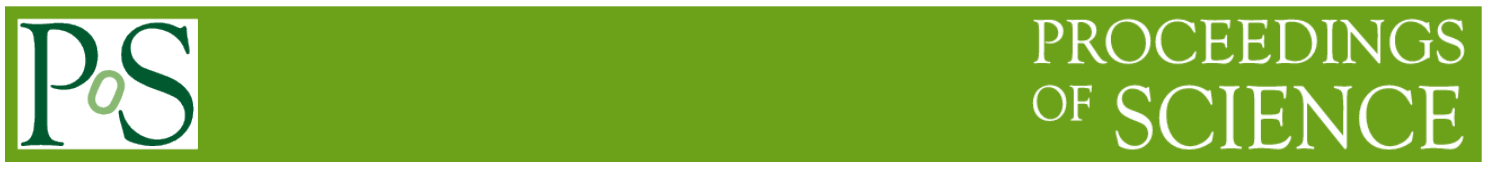

\title{
The NUCLEON Space Experiment Preliminary Results
}

\author{
A. Turundaevskiy ${ }^{\mathrm{a} 1}$, E.Atkin ${ }^{\mathrm{a}}$, V.Bulatov ${ }^{\mathrm{c}}$, V.Dorokhov ${ }^{\mathrm{c}}$, N.Gorbunov $^{\mathrm{d}}$, S.Filippov $^{\mathrm{c}}$, \\ V.Grebenyuk ${ }^{\mathrm{d}}$, D.Karmanov ${ }^{\mathrm{a}}$, I.Kovalev ${ }^{\mathrm{a}}$, I.Kudryashov' ${ }^{\mathrm{a}}$, M.Merkin ${ }^{\mathrm{a}}$, A.Pakhomov ${ }^{\mathrm{a}}$, \\ A.Panov ${ }^{\mathrm{a}}$, D.Podorozhny ${ }^{\mathrm{a}}$, D.Polkov ${ }^{\mathrm{c}}$, S.Porokhovoy ${ }^{\mathrm{c}}$, V.Shumikhin ${ }^{\mathrm{b}}$, \\ L.Sveshnikova $^{\text {a }}$, A.Tkachenko ${ }^{d}$, L.Tkachev ${ }^{d}$, M.Torochkov $^{a}$, O.Vasiliev $^{a}$, A.Voronin $^{\mathrm{a}}$ \\ ${ }^{a}$ Skobeltsyn Institute of Nuclear Physics, Moscow State University, Moscow, 119991, Russia \\ ${ }^{b}$ National Research Nuclear University “MEPhI”, Moscow, 115409, Russia \\ 'SDB Automatika, Ekaterinburg, 620075, Russia \\ ${ }^{d}$ Joint Institute for Nuclear Research, Dubna, 141980, Russia \\ E-mail: turun1966@yandex.ru
}

\begin{abstract}
The NUCLEON satellite experiment is designed to investigate directly the energy spectra of cosmic-ray nuclei and the chemical composition $(\mathrm{Z}=1-30)$ at energy range $100 \mathrm{GeV}-1000 \mathrm{TeV}$. The satellite has been launched in 26 December 2014. The planned exposition time is more than 5 years. The first preliminary results are presented including charge distributions and all particles energy spectra.
\end{abstract}

Frontier Research in Astrophysics - II

23-28 May 2016

Mondello (Palermo), Italy

${ }^{1}$ Speaker 


\section{Introduction}

The NUCLEON satellite experiment is designed to investigate directly, above the atmosphere, the energy spectra of cosmic-ray nuclei and the chemical composition from 100 $\mathrm{GeV}$ to $1000 \mathrm{TeV}$ (before the "knee"). The additional aim is the cosmic-ray electron spectrum measurement (from $20 \mathrm{GeV}$ to $3 \mathrm{TeV}$ ).

The "knee" energy range $10^{14}-10^{16} \mathrm{eV}$ is a crucial region for the understanding of the cosmic-ray, acceleration and propagation in the interstellar medium. It is important to obtain more data with elemental resolution. The "knee" area is interesting for astrophysics.

New experiments over a wide charge and energy range are needed. The satellite was launched in 26 December 2014. This mission is aimed at clarifying the essential details of cosmic-ray origin in this energy interval: number and types of sources, identification of actual nearby sources, the investigation of the mechanisms responsible for the knee. Specific features of the NUCLEON instrument are a relatively small thickness and a small weight. A special method of energy determination by the silicon tracker was developed for this case. In this paper we present a design of the instrument, some results of accelerator beam tests in terms of charge and energy resolution. The evidences of the capability of the apparatus to achieve the declared aims are presented also.

\section{The NUCLEON design}

The NUCLEON device [1,2] was designed and produced by a collaboration of SINP MSU (main investigator), JINR (Dubna) and some other Russian scientific and industrial centers. It is placed now on board of the RESURS-P №2 satellite. The spacecraft orbit is a Sun-synchronous one with inclination 97.276 and a middle altitude of $475 \mathrm{~km}$.

The effective geometric factor is more than $0.2 \mathrm{~m}^{2} \mathrm{sr}$ for the KLEM (Kinematic Lightweight Energy Meter) system and near $0.06 \mathrm{~m}^{2} \mathrm{sr}$ for the calorimeter. The surface area of the device is equal to $0.25 \mathrm{~m}^{2}$. The charge measurement system must provide resolution better than the 0.3 charge unit.

The NUCLEON apparatus includes different units based on silicon and scintillator detectors. The charge measurement system consists of the 4 pad silicon detectors layers. The KLEM energy measurement system includes 6 silicon microstrip detectors interleaved with thin tungsten layers. The trigger system consists of the 6 scintillator layers. The calorimeter includes 6 silicon microstrip detectors too.

The set of data obtained by all detectors can be considered as the image of the event. The event example is presented in fig.1.

Four charge detector layers are presented in the left top corner of the figure. Circles correspond to worked pads. The side projections of events are presented for silicon (right) an scintillator (bottom) detectors.

These data are used for analysis. 


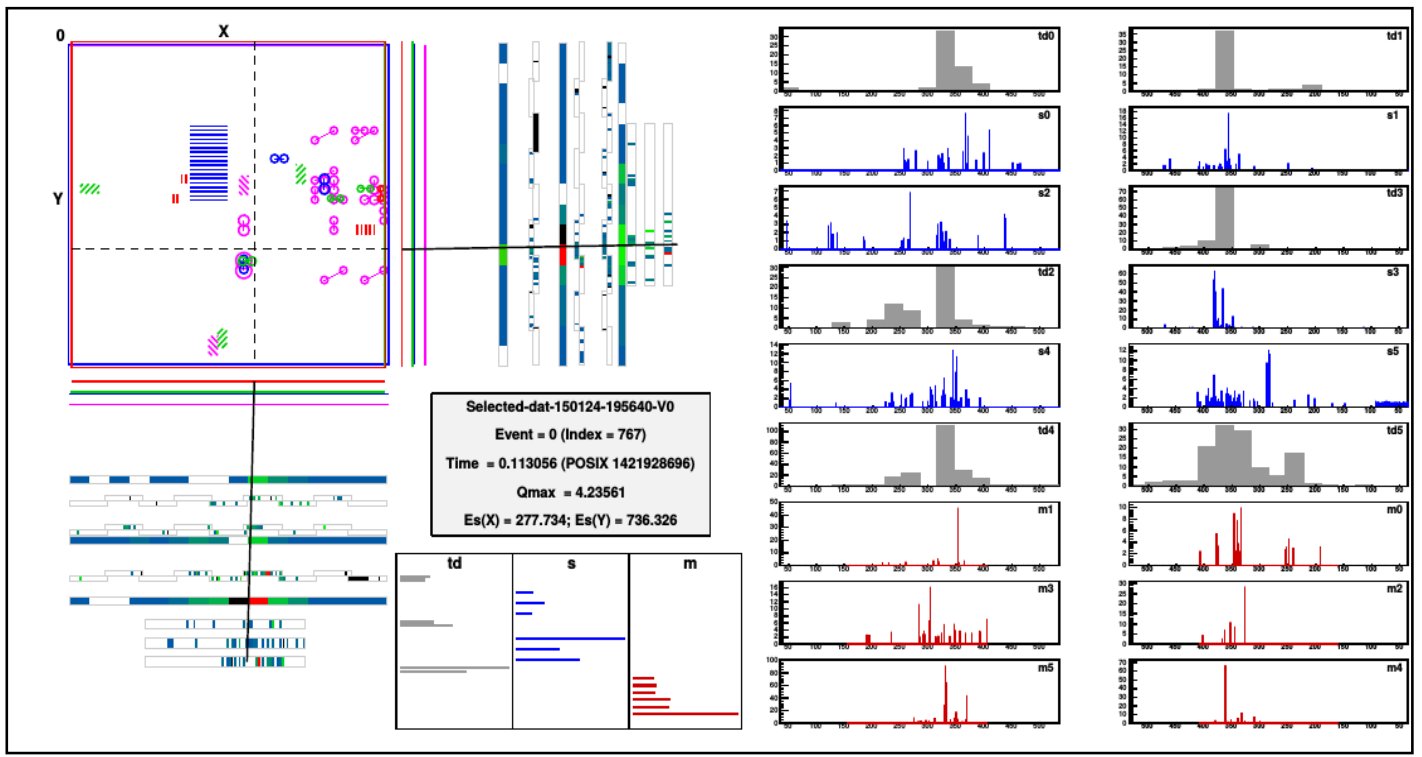

Figure 1: The image of the event. The boron nucleus initialized the shower

\section{The trigger system}

The trigger system parameters were optimized by the first stage of the space experiment. This system is aimed to generate necessary trigger signals to register the event. Each of the six trigger planes consists of the 16 scintillator strips. The light signals from the strips are collected by the wavelength shifting fibers (WLS) to photomultiplier tubes (PMT). The dead time is equal to $200 \mathrm{~ns}$.

Selection criteria include signal registration for all 6 layers. Different thresholds are used for different layers according to the shape of showers.

The trigger operational mode is determined by 6 thresholds of comparators. A single-channel PMT detects scintillation light from a layer. The signal arrives to the comparator. The trigger is given if at the same time not less than in $\mathrm{N}$ layers the signal exceeded the set thresholds.

The trigger system is intended for cosmic ray particles selection. To determine charge and energy it is neccessary to select particles transversed detectors. Electromagnetic component can be selected only for particles registered by the calorimeter. For hadrons the shower must be initiated by the first inelastic interaction in the carbon target. The trigger criteria define the energy threshold and measurements rates too.

\section{The charge measurements}

The charge detector system is designed for precision measurement of the primary particle charge and consists of four thin detector layers of $1.5 \times 1.5 \mathrm{~cm}$ silicon pads. Each readout channel is used for the two pads to decrease number of channels. Signals of two pads from different parts of detector are summed. Probability of simultaneous registration of two particles is 
negligible. Charge measurement readout chips CR-1 have a dynamic range $\sim 1000$ mip. The charge measurement system must provide resolution better than the 0.3 charge unit according to results of simulation and beam tests.

Microstrip detectors signals are used to reconstruct shower axis. Then charge detector pads near this axis are selected.

The system for determining the charge consists of four layers of silicon detectors composed of separate pads. Information on the incident particle charge is collected from each array independently of the others, which guarantees a higher accuracy of measurements than that achieved by a single array. Various algorithms of particle charge reconstruction were worked out. The calibration curves were obtained for all channels.

The charge spectra of the four detectors were matched using the rank statistics method [3]. For each recorded event four charges were measured by the four detectors and arranged in ascending order (regardless of the detector to which a particular charge corresponded). The next step was to determine the charge that is second in magnitude, and this value was used as the estimate for the charge. It should be noted that the rank statistics method provides better results than mere averaging of values, since fluctuations of the ionisation losses have a sharply asymmetrical form, as opposed to the standard distribution of errors. This method decreases errors caused by nuclear spallation and secondary particles generation in the detector.

The charge measurements were tested by ion beams [3]. In the Charge Measurement System there are peaks from different nuclei up to $Z=30$ with reasonable separation. The channel gain in electronics of the charge measurement system is not perfectly linear, the real signal from the heavy nuclei $(\mathrm{Z}>25)$ is higher than it should be from the standard dependence of the signal on the square of nuclear charge. The results of measurements were used for the charge reconstruction for the sateliite experiment.

The charge distribution obtained by the first stage of the NUCLEON satellite experiment is presented in fig.2. The preliminary analysis provides charge accuracy near $0.2-0.3$. We took into account different characterisics of silicon detectors.

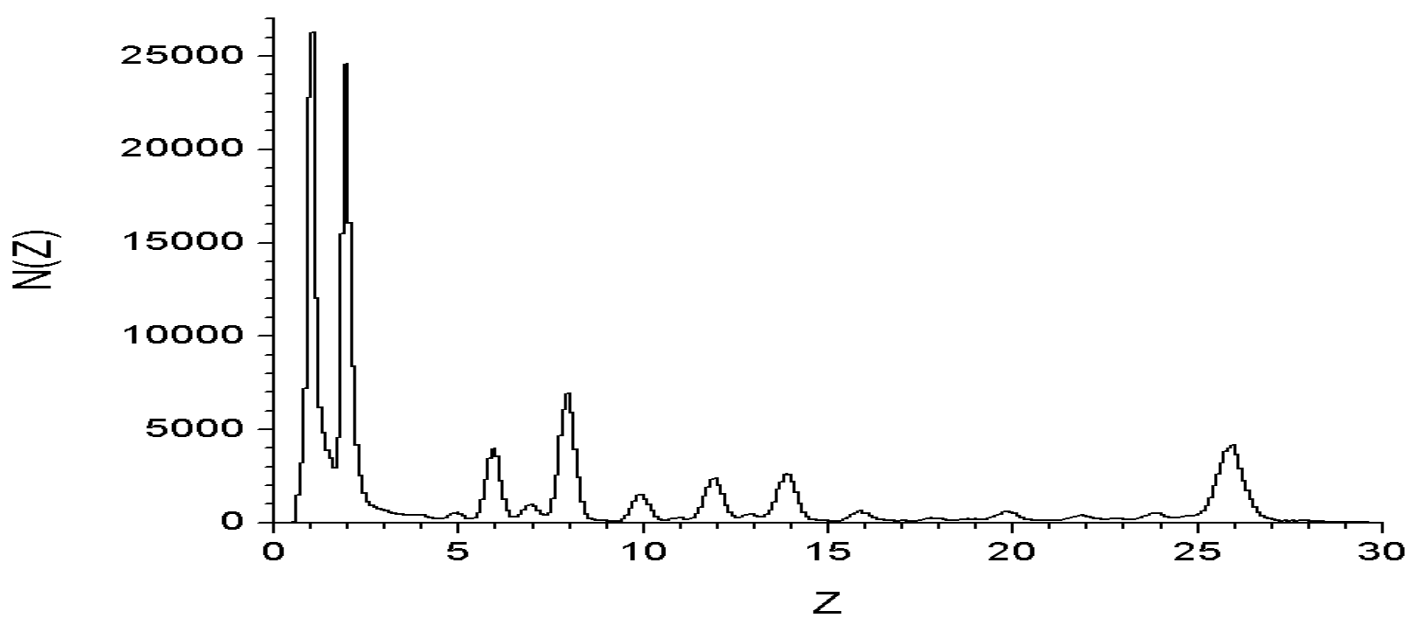

Figure 2: Preliminary charge distribution obtained by the NUCLEON experiment 
It is possible to improve resolution of measurements by means of new analysis algorithms. The strict selection of events was used to find beryllium and boron peaks. The nuclei registered by all track detectors including the calorimeter were selected. Moreover the events with small difference between charge signals in differerent detector layers were selected. The charge distribution for selected events is showed in fig.3.

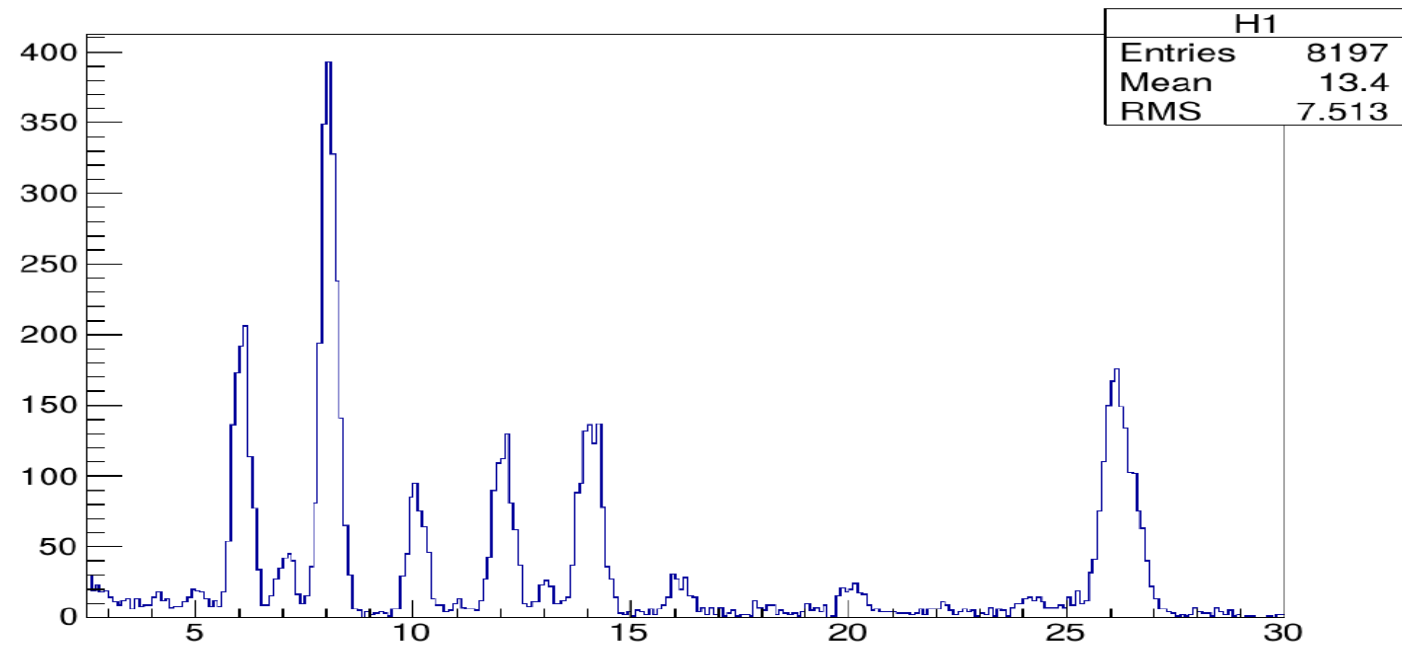

Figure 3: Charge distribution obtained for selected events

\section{Energy measurements}

The energy measurement system is based on KLEM technique [1]. Tungsten layers significantly increases the number of secondary particles and therefore improves the accuracy of a primary particle energy determination. The pitch of microstrips is equal to $484 \mu \mathrm{m}$. Every strip is connected to its own readout channel. The perpendicular strip orientation makes it possible to perform analysis for each (X and $\mathrm{Y}$ ) direction independently and improve the primary particle energy resolution.

The microstrip detectors can register many charged particles per strip. The signal is proportional to the strip ionisation or the number of single-charged particles. Thus S-estimator is defined as:

$$
S=\Sigma I_{k} \ln ^{2}\left(H / 2 x_{k}\right)
$$

where $x_{k}$ is a distance between the shower axis and the strip $k, I_{k}$ is a signal in the strip $k$.

The calibration curves were calculated for different components including protons, helium, carbon, sulfur, iron nuclei by means of simulation. The interpolation was applied to calculate calibration parameters for other nuclei. The energy dependence of the registration efficiency was determined too. These results were used to reconstruct primary energy spectra for the satellite experiment data.

The charge of the registered particle was determined by charge measurement system. The rank statistics was applied. The energy was calculated for every event by calibration curves. 
The energy spectra were normalized to the geometric factor, exposition time and the registration efficiency. The all particles spectrum was determined by summation of different nuclei spectra.

Another energy measurement system is based on traditional calorimeter technique for showers transversed the ionization calorimeter. Aperture events were selected. The deposited energy was determined by summation of all signals in the calorimeter microstrip detectors. Cascade curves were reconstructed by means of inerpolation.

The both methods are used for the cross-calibration. The energy was determined by two methods ( $E_{\text {KLEM }}$ and $E_{\text {MIC }}$ ) for different events. The correlation of energy deposited in the calorimeter and $\mathrm{S}$ estimator is presented in fig.4.

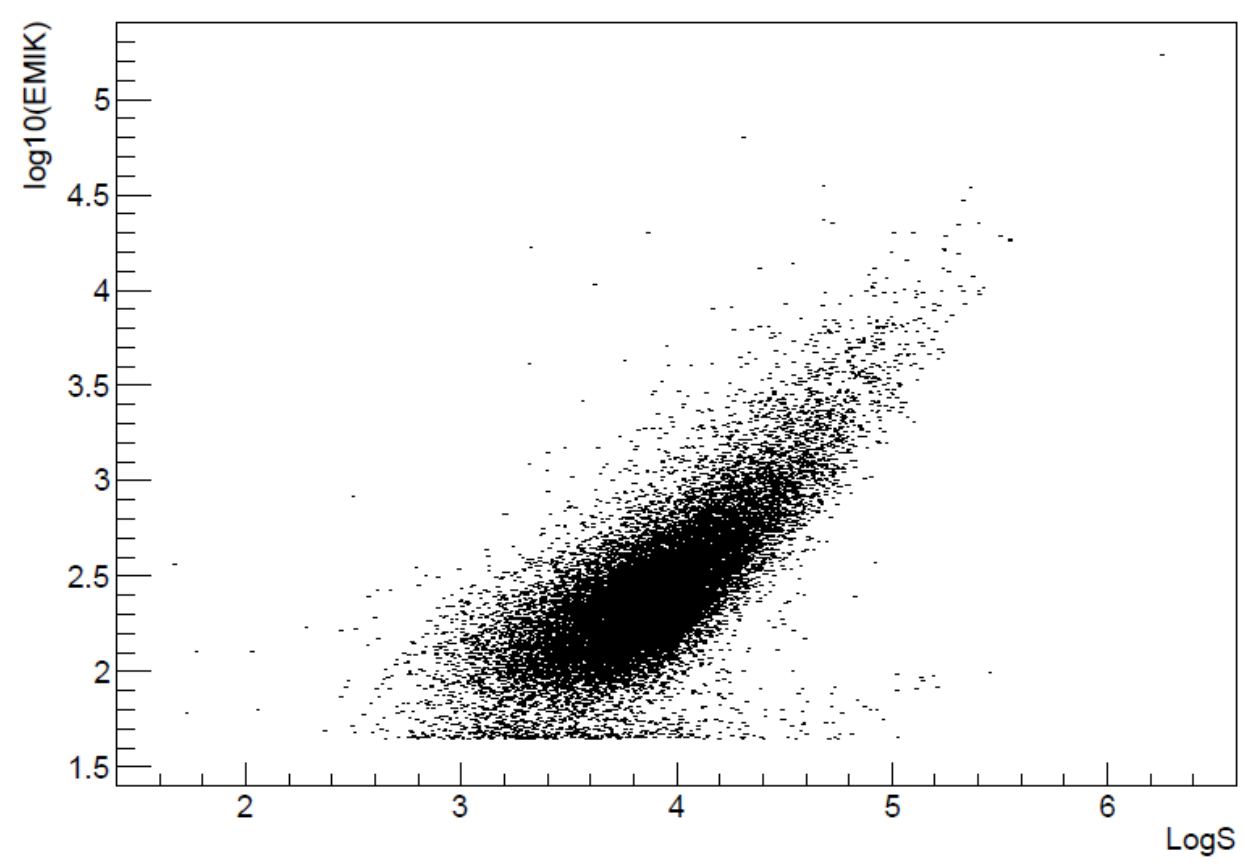

Figure 4: Calorimeter energy deposition vs S estimator

The Pearson product-moment correlation coefficient for $\mathrm{E}_{\mathrm{KLEM}}$ and $\mathrm{E}_{\mathrm{MIC}}$ is equal to 0.82 . The distribution of ratio $\mathrm{E}_{\mathrm{KLEM}} / \mathrm{E}_{\mathrm{MIC}}$ is presented in fig. 5 for primary protons and helium nuclei.

Energy values determined by two methods are close. The middle ratio $\mathrm{E}_{\mathrm{KLEM}} / \mathrm{E}_{\mathrm{MIC}}$ is equal to 0.87 . It is significantly less than measurement errors. 


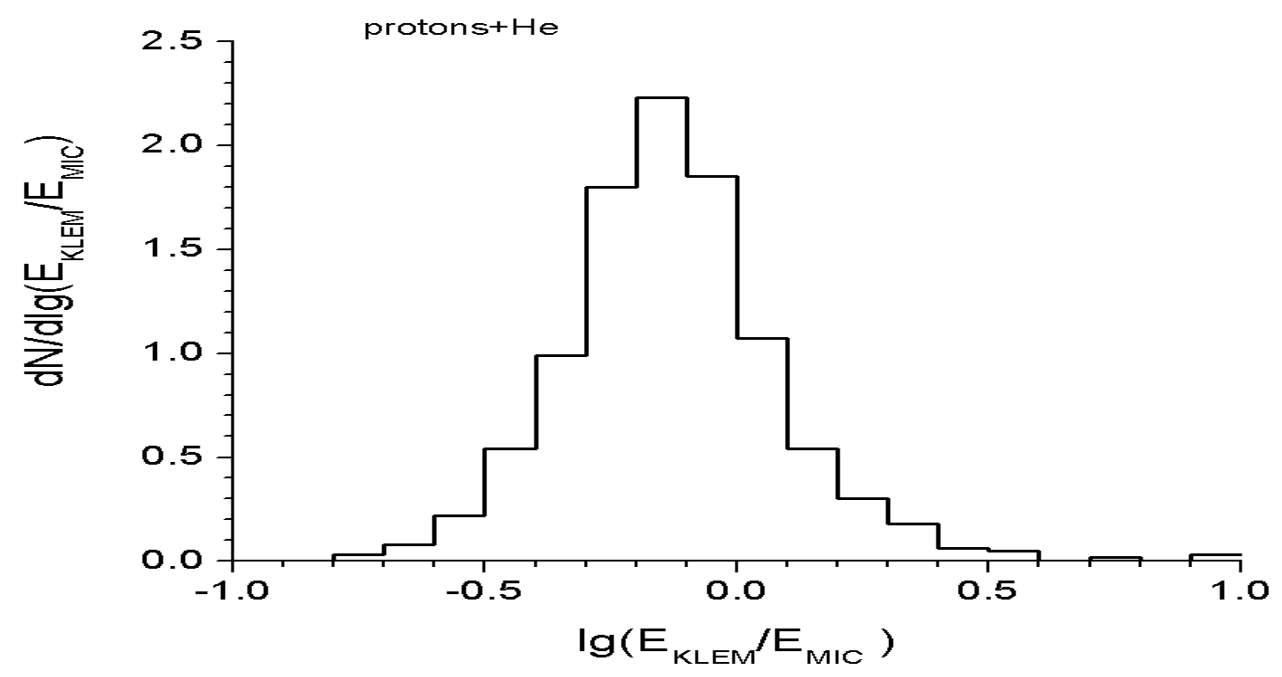

Figure 5: The distribution of ratio $\mathrm{E}_{\mathrm{KLEM}} / \mathrm{E}_{\mathrm{MIC}}$

The preliminary reconstructed all particles energy spectra are presented in fig.6. These spectra are obtained by means of KLEM and MIC techniques.

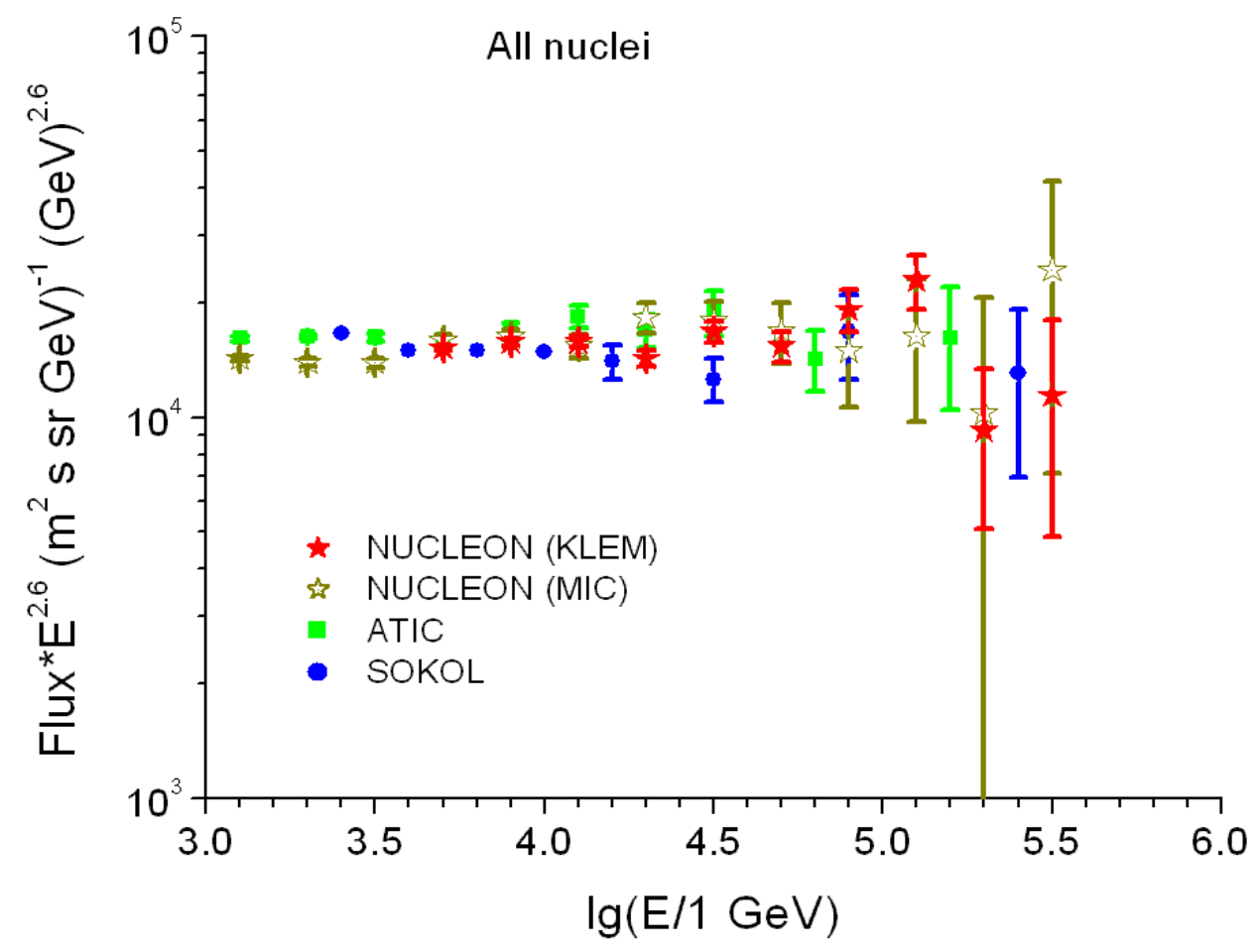

Figure 6: Preliminary reconstructed all particles energy spectra obtained by the NUCLEON space experiment by means of KLEM and MIC techniques 
At high energies the spectrum is close to power law with exponent near 2.6. The spectra obtained by KLEM and calorimeter techniqes are closed.

\section{Summary}

The NUCLEON device was designed and tested. The RESURS-P satellite was launched 26 December 2014. The expected performance is confirmed by simulation, beam tests and first space experiment results. All scientific objectives are achievable. We obtain and analyze the first data. The preliminary charge distributions and energy spectra are reconstructed.

\section{References}

[1] E. Atkin, V. Bulatov, V. Dorokhov et al., The NUCLEON space experiment for direct high energy cosmic rays investigation in $\mathrm{TeV}-\mathrm{PeV}$ energy range, Nucl. Instr. and Meth. in Phys. Research $\mathbf{A 7 7 0}$ (2015) 189

[2] E. Atkin, V. Bulatov, V. Dorokhov et al., The nucleon space experiment. EPJ Web of Conferences, 105:01002-p1-01002-p4 (2015).

[3] G.Voronin, V.Grebenyuk, D.Karmanov et al., Testing a Prototype of the Charge-Measuring System for the NUCLEON Setup, Instruments and Experimental Techniques 50, (2007) 187 